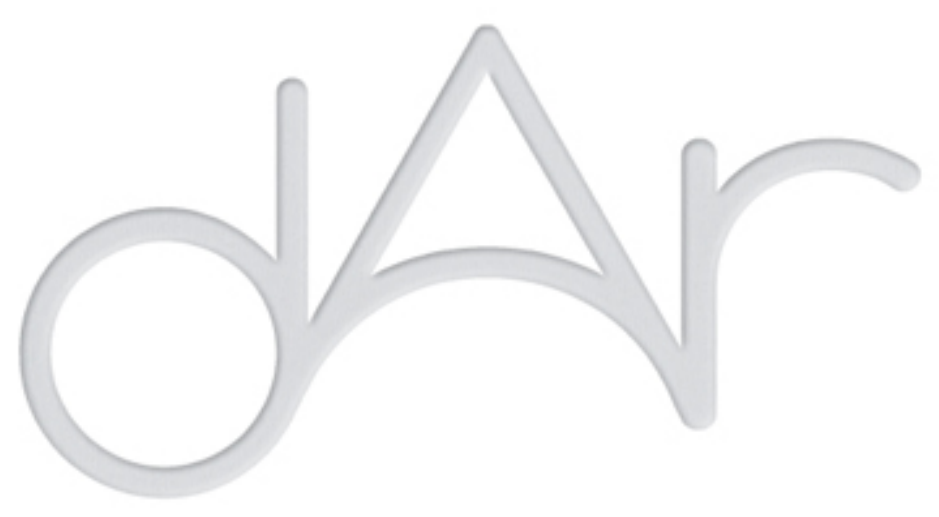

\title{
Patrimoine en fête: evénementiel et développement culturel dans les zones de montagnes: illustration par la fête du bijou d'Ath-Yenni en Algérie
}
Autor(es):
Berbar, Mouloud; Souidi, Rosa Aknine
Publicado por: Imprensa da Universidade de Coimbra
URL
persistente:
URI:http://hdl.handle.net/10316.2/44437
DOI:
DOI:https://doi.org/10.14195/2182-844X_5_16
Accessed : $\quad$ 26-Apr-2023 14:35:03

A navegação consulta e descarregamento dos títulos inseridos nas Bibliotecas Digitais UC Digitalis, UC Pombalina e UC Impactum, pressupõem a aceitação plena e sem reservas dos Termos e Condições de Uso destas Bibliotecas Digitais, disponíveis em https://digitalis.uc.pt/pt-pt/termos.

Conforme exposto nos referidos Termos e Condições de Uso, o descarregamento de títulos de acesso restrito requer uma licença válida de autorização devendo o utilizador aceder ao(s) documento(s) a partir de um endereço de IP da instituição detentora da supramencionada licença.

Ao utilizador é apenas permitido o descarregamento para uso pessoal, pelo que o emprego do(s) título(s) descarregado(s) para outro fim, designadamente comercial, carece de autorização do respetivo autor ou editor da obra.

Na medida em que todas as obras da UC Digitalis se encontram protegidas pelo Código do Direito de Autor e Direitos Conexos e demais legislação aplicável, toda a cópia, parcial ou total, deste documento, nos casos em que é legalmente admitida, deverá conter ou fazer-se acompanhar por este aviso.

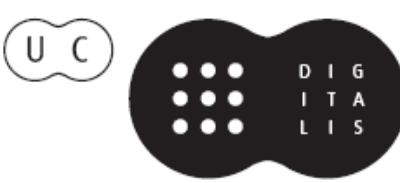



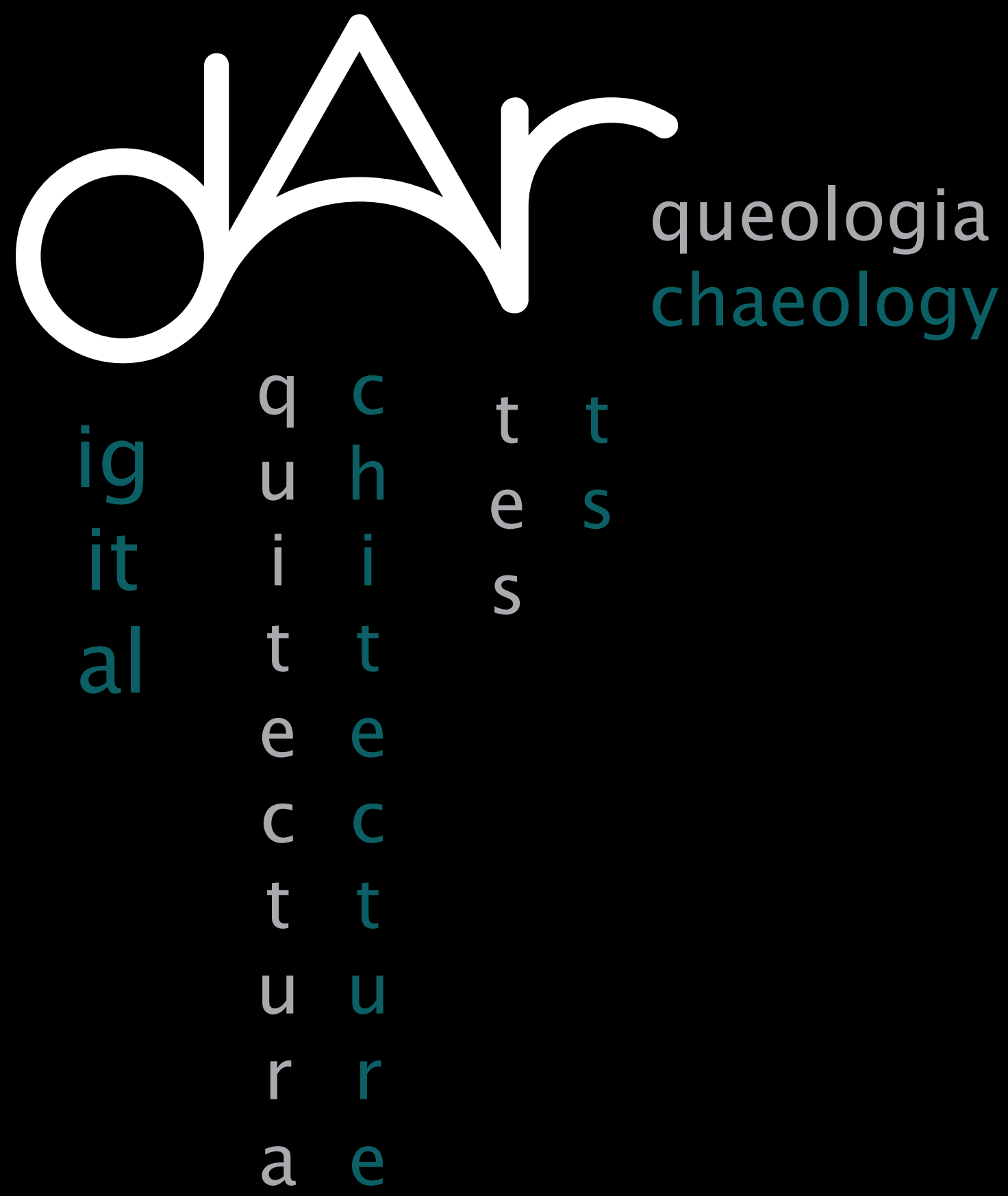

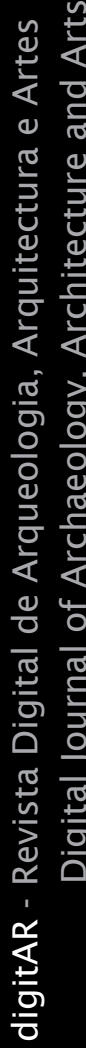




\title{
Patrimoine en fête: Evénementiel et développement CUlturel dans Les ZONES DE MONTAGNES. Illustration PAR LA FÊTE DU BIJOU D'ATH-YENNI en Algérie
}

\author{
Mouloud Berbar, Dr. Rosa Aknine Souidi \\ Université Mouloud MAMMERI de Tizi-Ouzou, Algérie.
}

\section{Résumé}

L'objet de notre article est d'analyser l'apport de l'événementiel sur la valorisation du patrimoine culturel immatériel dans les zones en difficultés. Pour ce faire, nous mettrons en relation la notion de l'événementiel avec celle des zones de montagnes. Ces dernières se caractérisent par des ressources matérielles et immatérielles qui façonnent les activités des populations locales et conditionnent la réussite des manifestations culturelles de valorisations patrimoniales dans ces régions. A travers le cas de la fête du bijou d'AthYenni nous tenterons de caractériser l'apport de l'événementiel dans le renforcement de la spécificité du bijou traditionnel et dans l'impulsion d'un développement culturel local.

Mots clés : Evénementiel culturel, zones de montagnes, développement culturel local, fête du bijou, Ath-Yenni.

\section{Introduction}

Le développement territorial durable, ne peut se réaliser uniquement sur la base de ressources génériques. L'approche territoriale de l'économie (Collitis et Pecqueur; 2004 ; Pecqueur, 2006 ; Courlet, 2008 ; François et al. 2009) stipule que se sont les ressources spécifiques qui déterminent un développement économique par le bas. Cette démarche est sensée suivre une logique de spécification sur tous les niveaux de la ressource à savoir l'identification, la construction, l'activation et la valorisation.

Le patrimoine culturel immatériel se caractérise par sa spécificité, qui tient à l'originalité des ressources qu'il mobilise. Depuis les années 1970, il est confronté aux effets de la mondialisation et aux transformations qu'elle a 
induit. Dès lors, des initiatives de valorisation sont engagées pour assurer sa sauvegarde et lui redonner une place prépondérante dans l'activité économique notamment au niveau local. L'événementiel constitue l'une des formes de valorisation de ce patrimoine.

L'événementiel culturel a connu un essor depuis quelques années en Algérie ; des fêtes et festivals locaux sont organisés dans les villes comme dans les villages tout au long de l'année. Ceci s'explique non seulement par une volonté de promouvoir les ressources locales mais d'impulser un développement culturel, notamment dans des zones de montagnes, défavorisées du point de vue d'accès à la culture. La fête de bijoux d'AthYenni organisée chaque année est un exemple dans le domaine.

L'objet de notre travail est double. D'une part nous analyserons l'impact de cette fête d'abord sur le maintien du cachet Kabyle et local du bijou en argent (labellisation culturelle), puis sur le développement culturel du territoire, d'autre part nous tenterons de savoir qu'est ce que les zones de montagnes offrent-elles de spécifique à l'événementiel pour assurer à partir du patrimoine culturel immatériel un développement culturel durable? Pour analyser l'apport de la fête du bijou dans le développement culturel dans la région d'Ath-Yenni, nous avons réalisé une enquête de terrain à base de guides d'entretiens auprès des artisans et des organisateurs de la fête du bijou d'Ath-Yenni lors des deux dernières éditions (2015 et 2016). Pour discuter les résultats obtenus, nous mobiliserons une grille d'analyse portant sur les ressources territoriales. Ce choix est motivé par le fait que l'événementiel constitue un levier de valorisation et de spécifications des ressources territorialement ancrées.

Notre article se structure en trois parties, la première propose un retour sur la notion de l'événementiel culturel, la seconde caractérisera la fête du bijou dans le territoire d'Ath-Yenni en tant que zone de montagne. La dernière sera consacrée à l'analyse du rôle de la viabilité du territoire dans la valorisation du bijou d'Ath-Yenni par un événementiel, comme nous présenterons l'apport de la fête sur le développement culturel de la région.

\section{Evénementiel et savoir-faire traditionnels: quelle stratégie de valorisa- tion?}

\section{1. Un retour sur la notion de l'événementiel en théorie et dans la pra- tique algérienne}

L'origine de l'événementielle remonte au moyen âge et l'antiquité. En effet, des fêtes et foires sont perpétuellement organisées (Turbiez, 2010). 
Ces événements s'inscrivent dans le rituel et les mœurs des civilisations, ainsi, les sacres de rois étaient toujours célébrés pour renforcer et sacraliser leur pouvoir et marquer les esprits des populations. Ces événements ont pour objectif de divertir les populations et chercher une rupture avec leur vie quotidienne caractérisée par la difficulté et la misère. D'autres événements ont ensuite vu le jour et marqué l'histoire de l'événementiel, on peut citer la première exposition universelle (1851) dans le domaine de la culture, les jeux olympiques (1892) et la coupe du monde (1928) dans le domaine sportif.

L'événementiel s'est développé par la suite en fonction de la transformation et de l'évolution des besoins, ainsi il s'oriente vers autres finalités telles que la communisation et le marketing à partir des années 1970 avec l'émergence d'entreprises spécialisées dans l'événementiel (Babkine et Robier, 2011).

L'événementiel renvoie à une manifestation ou une animation, organisées dans un espace déterminé pour une durée donnée. Il s'agit d'une forme de communication visant à valoriser un potentiel matériel ou immatériel. "L'événementiel est le fait d'organiser une manifestation, de réunir dans un même lieu des individus ciblés pour une occasion précise et à un moment déterminé. » (Babkine et Robier, 2011, 2)

Miranda et Cermakova (2009), définissent l'événementiel en mettant en avant la notion de l'événement urbain festif (EUF) qui renvoie à «toutes sortes de manifestations liées au domaine de la culture et des loisirs ; il est organisé à une échelle urbaine ; il a la capacité d'attirer l'attention des acteurs territoriaux externes - régional, national, international - et de mobiliser la population interne d'un territoire par une rupture flamboyante avec le quotidien par l'originalité de sa mise en scène, sa temporalité éphémère et son ambiance festive maîtrisée ; il a pour principal objectif de susciter diverses dynamiques - de promotion, légitimation, engagement, coopération - autour de projets collectifs de développement économique, social et urbain sur le territoire de son déroulement. " (Miranda et Cermakova , 2009, 390) Ces événements ont donc pour objectifs de légitimer une ressource, sensibiliser les acteurs concernés et stimuler un engagement de toute la communauté dans l'organisation de l'événement. La notion l'événementiel est utilisée différemment selon le domaine concerné et l'objectif qui lui est assigné. L'événement s'exprime sous plusieurs formes et dénominations ; on distingue les festivals, les fêtes, les foires et les salons. La portée territoriale locale, nationale et internationale de ces manifestations complexifie d'avantage la possibilité 
d'avoir une définition précise et représentative de la notion en question. Ces formes ont des dénominateurs communs à l'instar de l'originalité et de la configuration en termes d'espace-temps. Un festival est ainsi considéré comme " une forme de fête unique, célébration publique d'un genre artistique dans un espace temps, réduit à périodicité annuelle » (Benito, 2002, 25).

Di Méo (2001), définit l'événementiel en faisant référence à la fête qui selon lui, renvoie à «la quintessence de toutes les formes festives. Elle trace un espace-temps aussi bref qu'intense » (Di Méo, 2001, 11). L'auteur met l'accent également sur la portée territoriale de la fête qui selon lui, elle renforce ses représentations sociales et intensifie son ancrage territorial. L'événementiel culturel est assimilé à des rencontres ayant trait au domaine de la culture, celles-ci sont circonscrites sur une durée déterminée et dans un espace donné. Il se présente sous l'une des formes que nous venons de citer. En théorie il existe une différence entre différentes formes ; le festival renvoie à une manifestation culturelle qui a pour objet l'exposition des œuvres artistiques (matérielles et immatérielles) dans une logique festive, par contre la fête est assimilée à une célébration partagée collectivement. De son côté, la foire est à caractère purement commercial.

Dans le sillage de la valorisation du patrimoine culturel matériel et immatériel, la distinction entre ces formes est confuse étant donné que les objectifs qui leur sont assignés sont similaires à savoir, la promotion, la valorisation et la sauvegarde de l'héritage ancestral.

Le choix de l'une ou de l'autre forme de l'événementiel diffère selon la politique envisagée par les autorités publiques. En effet "l'emploi et l'usage permanent des mots «festival», «fête», «festif», «événement» et leur amalgame fréquent par les collectivités locales et les médias tiennent au fait qu'ils sont englobés dans un même registre de l'action publique culturelle. Fêtes, festivals, journées (du patrimoine, de l'environnement...) deviennent parfois équivalents » (Garat, 2005, 266).

En Algérie, la distinction entre les formes de l'événementiel culturel répond à une logique institutionnelle. Les formes «festival » et «salon » concernent les manifestations organisées et financées par l'acteur public auquel est rattaché le patrimoine culturel, à savoir la direction de la culture et la direction du tourisme et de l'artisanat.

Selon la logique institutionnelle de l'Algérie, « le festival » est à caractère thématique, réservé aux événements qui ont une portée locale ou régionale, on peut citer le festival culturel local de la poterie de Maâtkas. "le salon » est assimilé aux manifestations nationales et internationales, il est d'une 
dimension plus large et ne cible pas un patrimoine déterminé (les salons nationaux de l'artisanat traditionnel organisés annuellement dans chaque wilaya du pays, le salon international de l'artisanat traditionnel tenu une fois par an à Alger, etc.).

A contrario des deux formes précédentes, la fête est plutôt l'émanation de la communauté locale, elle est organisée par les associations d'artisans, les comités des villages, les acteurs du métier (artisans, agriculteurs) en collaboration avec les autorités publiques. On peut citer la fête de la poterie du village Ait Kheir, la fête du bijou d'Ath-Yenni et la fête de la figue du village Lemsella.

Ces différentes partagent une finalité publicitaire (une logique de promotion) selon laquelle l'événementiel cible des acteurs territoriaux et attire leur attention sur des éléments jusque là méconnus (Chouchan et al, 2000 ; Miranda et Cermakova, 2009). Toutes ces formes sont marquées par le caractère collectif de leur déroulement, elles engagent un ensemble d'acteurs territoriaux. On tentera dès lors de caractériser l'engagement communautaire qui règne dans ces événements et qui constituent des facteurs clés de spécification et de sauvegarde du patrimoine culturel immatériel par l'événement en question. Nous procéderons par l'exemple de la fête du bijou d'Ath-Yenni pour analyser l'apport de la communauté de cette région dans l'organisation et la spécification des deux ressources à savoir la fête et le bijou.

1.2 L'événementiel culturel : Qu'elle trajectoire de valorisation du bijou d'Ath-Yenni pour quelle stratégie de développement local durable?

L'objectif principal de l'événementiel est de promouvoir la spécificité du patrimoine culturel immatériel. Grâce à leur ancrage territorial, les savoir-faire traditionnels sont caractérisés par une spécificité que les initiatives de valorisation doivent renforcer, afin d'inscrire la trajectoire de cette ressource dans une logique de spécification.

La réussite de l'événementiel, quelque soit sa forme, tient à sa capacité à renforcer la spécificité du savoir-faire traditionnel. Notre analyse se basera sur la notion de ressources territoriales spécifiques et les trajectoires de ressources possibles afin de positionner l'apport de la fête du bijou d'AthYenni dans le renforcement du cachet authentique et spécifique du bijou. La notion de ressource est relative, elle dépend de l'interprétation qu' on lui accorde. «Par ressource territoriale on comprend une caractéristique d'un territoire issue de l'activité humaine intentionnelle et non-intentionnelle et qui peut servir comme intrant dans le processus de production » (Samaganova et Samson, 2007, p3) 
Collitis et Pecqueur (2004) proposent une distinction entre ressources spécifiques et génériques. Les ressources spécifiques sont en relation avec le vécu du territoire et son histoire, elles résultent «d'une accumulation de mémoire, d'un apprentissage collectif cognitif... La production de telles ressources résulte de règles, de coutumes, d'une culture élaborées dans un espace de proximité, géographique et institutionnelle à partir d'une forme d'échange distincte de l'échange marchand : la réciprocité.» (Collitis et Pecqueur, 2004, 58). Ces ressources sont propres à un territoire donné, elles sont façonnées par son environnement.

A partir de cette définition, on peut dire que le bijou d'Ath-Yenni peut être assimilé à une ressource spécifique. Il s'agit d'une activité qui remonte au $14{ }^{\text {ème }}$ siècle (Donsimoni et al. 2010) sa spécificité et son originalité tient d'un côté aux ressources utilisées notamment pour la décoration et le design, et de l'autre, aux différentes gammes du bijou et au savoir-faire ancestral qui se transmet de génération en génération.

Le défi de l'événementiel se situe dans l'inscription de la ressource dans une logique de spécification, nous allons ainsi présenter les trajectoires possible d'une ressource territoriale et démonter le rôle d'opérateur de la ressource que joue l'événementiel.

Le caractère spécifique et générique de la ressource est lié à la fois aux dynamique de construction de cette dernière et aux modes de sa valorisation. François et al., (2009), proposent une matrice des fromes de trajectoires de la ressource en procédant à un croisement entre les modes de construction et de valorisation de la ressource, selon qu'ils soient spécifiques ou génériques, tel qu'il est présenté dans la figure ci-dessous.

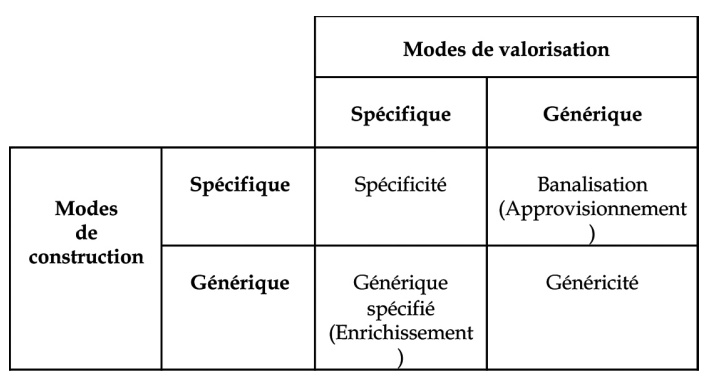

Tab. 1 - Matrice des trajectoires possible de la ressource. Source : François et al, 2009
Selon François et al., (2009), ce croisement donne lieu à quatre trajectoires de la ressource qu'on peut regrouper en deux types:

Les dynamiques continues: ce sont des dynamiques dont la trajectoire assure un continuum du caractère de la ressource;

- $\quad$ Les dynamiques modificatrices: qui renvoient à des dynamiques qui modifient le caractère de la ressource. 


\section{2. 1. Les dynamiques continues de la ressource}

\subsubsection{La spécificité}

Cetteformeapparaitsuiteàl'application d'unmodedevalorisation spécifique sur une ressource spécifique. Cette trajectoire permet à la ressource de maintenir son caractère spécifique et l'inscrit dans une logique de renouvellement tout en misant sur la sauvegarde de son identité et de son caractère patrimonial. Selon cette trajectoire, la sauvegarde de la spécificité d'une ressource est conditionnée par la prise en compte de son niveau externe, il s'agit du marché (la demande) et de la filière de l'activité où sont exprimées les attentes des consommateurs et des acteurs de la filière; se sont des déterminants que les stratégies de valorisation prennent en compte. (François et al, 2009).

La fête du bijou d'Ath-Yenni permet de positionner le savoir-faire de bijouterie de cette région dans cette trajectoire, car elle répond à une construction originale et spécifique à base d'un produit local unique en l'occurrence le bijou traditionnel.

\section{2. 1. 2. La généricité}

Il s'agit d'une forme dans laquelle les modes de valorisation et de construction sont réalisés suivant une démarche générique. Dans ce cas, on a une ressource générique, valorisée par des mécanismes génériques, répondus et connus dans d'autres espaces. Dans ce cas, les spécificités du territoire ne sont pas mises en avant. (François et al, 2009).

Dans certains cas, les savoir-faire traditionnels subissent cette trajectoire notamment avec la standardisation des procédés de production et des programmes de formations dispensés sur des territoires différents. Le caractère esthétique d'un produit diffère d'une région à une autre, voire même d'un village à un autre. Il est recommandé que cette originalité soit prise en compte par les centres de formation aux métiers d'art et à l'artisanat dans la mesure où, il est souhaité que leurs programmes soient territorialisés afin de préserver la spécificité du savoir-faire traditionnel.

\section{2. 2. Les dynamiques modificatrices de la ressource}

\section{2. 2. 1. La spécification/Générique spécifiée (Enrichissement)}

Dans ce cas, la ressource est soumise initialement à une construction 
générique, elle subie une valorisation spécifique. Il s'agit d'une trajectoire d'enrichissement d'une ressource générique par des modes de valorisation spécifiques, la diversification du produit par des stratégies de marketing, entre dans ce cas. La labellisation constitue l'un des modes de valorisation permettant de spécifier une ressource générique en lui permettant un ancrage territorial et une construction d'un marché local (François et al, 2009).

\section{2. 2. 2. La banalisation (appauvrissement)}

C'est une trajectoire issue d'un mode de valorisation générique appliquée sur une ressource spécifique (François et al, 2009). Une démarche qui mène à une dilution de la spécificité de la ressource et donc à sa banalisation. A l'exemple d'un produit local spécifique qui sera soumis à des normes de production a-territoriales. L'exigence de spécification peut faire appel à des normes de valorisation générique impactant négativement la ressource en appauvrissant sont caractère spécifique.

La fête du bijou d'Ath-Yenni permet de renforcer la durabilité et la perpétuité du savoir-faire traditionnel en l'inscrivant dans une dynamique continue qui est « la spécificité ». Cette trajectoire permettrait d'asseoir une politique de développement durable autour du savoir-faire traditionnel de bijouterie.

2. La fête du bijou d'Ath-Yenni : quels déterminants territoriaux pour une valorisation par l'événementiel dans une zone de montagne?

\section{1. Zones de montagnes/zones en difficultés: quelles ressources pour le patrimoine culturel immatériel?}

Les zones de montagnes figurent parmi les zones en difficultés, elles désignent " des zones de faibles capacités, dont les milieux naturels sont hostiles ou contraignants... parfois peu accessibles et plus faiblement urbanisées. Les espaces ruraux des zones difficiles sont généralement caractérisés par des niveaux élevés de pauvreté, des taux d'équipement et des services moins présents qu'ailleurs. Ce sont des régions difficiles à mettre en valeur par les modes de développement conventionnels, mais dotées de paysages rares, et naturellement riches en biodiversité. » (Requier-Desjardins, 2013, p113).

La difficulté des zones de montagnes, des oasis et des zones arides tient à leur relief défavorable qui fait éloigner les projets des grands investisseurs. En effet, le modèle économique standard basé sur les grandes entreprises intensives en technologie ne peut tenir route dans ces zones. Se sont des régions à faible rentabilité pour ces entreprises. Leur dotation en ressources matérielles et immatérielles, génériques, spécifiques et endémiques qui 
peuvent être activées et valorisées, les met au premier rang des régions qui peuvent enclencher un développement inclusif, par le bas. Elles sont au centre des analyses de l'économie territoriale.

Des exemples concrets en Tunisie, au Maroc et en Algérie, ont démontré que la difficulté de ces zones était une sorte de "ressource » pour les populations locales ; ainsi dans leur processus d'adaptation, les populations ont réussi à construire des ressources immatérielles spécifiques, à l'instar des savoirs, savoir-faire et liens sociaux qui sont mobilisés quotidiennement pour valoriser les ressources locales de ces régions. Ces démarches ont donné naissance à des systèmes de production traditionnels parmi lesquels ont peut citer l'artisanat traditionnel.

\section{2. Ath-Yenni: Un exemple de zones de montagnes}

Ath-Yenni est une commune qui se situe dans la zone de haute montagne de la Kabylie du Djurdjura. Elle se caractérise par un terrain accidenté et se situe à 850 mètres d'altitude à $35 \mathrm{~km}$ au sud-est du chef de la wilaya de TiziOuzou. Elle est d'une superficie de $32,25 \mathrm{~km}^{2}$.

La commune est composée de sept villages: Ait Larbaa, Ait Lahcène, Taourirt Mimoun, Taourirt L'Hadjadj, Agouni Ahmed, Tigzirt, Tansawt. Les photos ci-dessous démontrent le relief accidenté de la région.

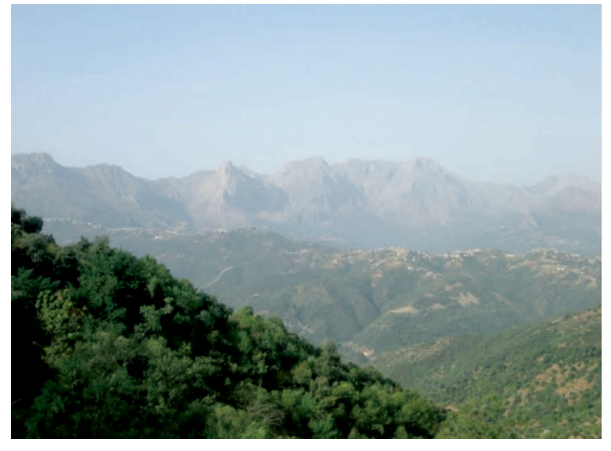

Fot. 1 - Une partie des villages d'Ath Yenni. Source : Photos prises par les auteurs

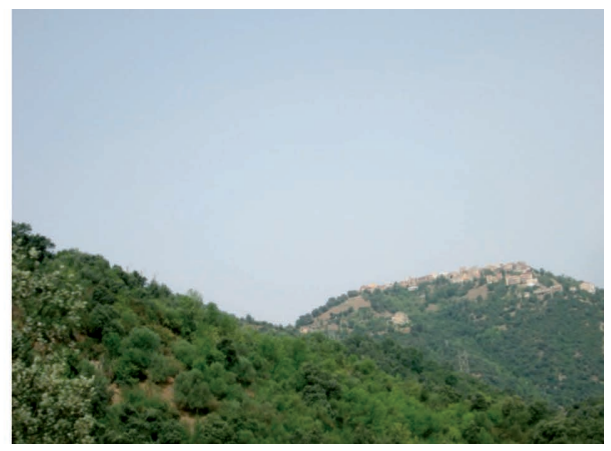

Fot. 2 - Village Taourirt Mimoun Source : Photos prises par les auteurs

Les villages d'Ath-Yenni se caractérisent par leur spécialisation dans le savoir-faire de bijouterie. Certains des artisans ont choisi de s'installer ailleurs, dans les grandes villes pour se rapprocher du marché (Tizi-Ouzou, Boumerdes, Alger, Constantine, Oran), d'autres ont choisi de perpétuer leur métier au sein de leur village. A l'origine le métier est masculin, par la suite, 
les femmes se sont mises à la production à domicile.

La production artisanale du bijou à Ath-Yenni est marquée par un travail de réseau composé d'artisans village et des autres villages de la région. Ce réseau trouve ses racines dans les rapports sociaux qui règnent dans chaque village tel est le cas dans tous les villages kabyles. Ces rapports se caractérisent par une réciprocité, des échanges et des initiatives communes aux bijoutiers de la région en matière d'approvisionnement collectif, d'échange d'information et de transmission de nouvelles techniques de production.

2.3 Présentation de la fête du bijou d'Ath-Yenni

La fête du bijou d'Ath-Yenni est perpétuée chaque année depuis sa création. En 2016 elle était à sa $13^{\text {ème }}$ édition. L'événement regroupe les bijoutiers d'Ath-Yenni, qui sont scindés en deux partie: ceux qui travaillent dans les villages d'Ath-Yenni et ceux qui se sont implantés dans d'autres régions (ville de Tizi-Ouzou, Alger, Bouira, Boumerdes, etc.). La diversité du savoirfaire bijoutier algérien est également valorisée par la présence des artisans des autres willayas à l'instar des bijoutiers de Tamanrasset, de Galma et de Batna.

La spécificité de la fête du bijou d'Ath-Yenni tient à son organisation qui regroupe l'ensemble des communautés villageoises d'Ath-Yenni. L'acteur organisateur de la fête du bijou est le Comité Communal des Fêtes (CCF), ce dernier est composé de commissions qui s'occupent de la restauration, l'hébergement, le transport, l'organisation, la sécurité et le sponsoring. Ce comité intègre d'autres acteurs organisateurs à savoir les comités de villages et l'association des bijoutiers d'Ath-Yenni. Les partenaires du CCF sont l'Assemblée Populaire Communale (APC) d'Ath-Yenni, la Direction de l'Aménagement du Territoire, du Tourisme et de l'Artisanat et la Chambre des Métiers et de l'Artisanat (CAM).

La fête s'étale sur une dizaine de jours et se déroule sur deux sites, il s'agit du collège Larbi MEZANI et la maison de jeune KEDDACHE Ali. Depuis son lancement, la fête connait une promotion au niveau national et international. Elle s'est intégrée dans les traditions rituelles de la région d'Ath-Yenni. Le nombre de visiteurs avoisinait 60000 en 2016, quant au nombre d'artisans, il a atteint 110 durant la fête de la même année.

L'événement est l'occasion pour promouvoir la culture locale des villages d'Ath-Yenni à travers le bijou. Chaque année la fête est placée sous un slogan, ce dernier a pour finalité de sensibiliser les acteurs territoriaux publics, privés et ceux du tiers secteur (à l'exemple des associations) face à la menace de disparition du métier, ainsi en 2011 elle était placée sous le slogan « De grâce, ne me laissez pas mourir ! » et en 2014 « Honneur à l'artisan bijoutier ». 
Ces slogans expriment également les difficultés des artisans et les exigences du savoir-faire bijoutier en tant que source de revenu pour les populations de la région ; dans ce sens, en 2012, «Le bijou d'Ath-Yenni, un art et une économie » était le slogan de la fête et en 2016 « Le bijou d'Ath-Yenni, héritage ancestral et exigences d'aujourd'hui ». Lors de la fête, les artisans profitent de la présence du ministre et des représentants de la direction du tourisme et de l'artisanat pour exprimer leurs difficultés et contraintes à titre de la cherté et de la rareté de la matière première ; ainsi, des mesures sont souvent prises par ces autorités pour y remédier.

La promotion de la bijouterie d'Ath-Yenni s'exerce également par l'organisation de conférences lors de fête, animées par des chercheurs et responsables des autorités publiques du secteur de l'artisanat. L'objectif consiste à sensibiliser les artisans par rapport aux politiques publiques ayant trait à l'artisanat traditionnel mais aussi à diffuser le caractère historique et identitaire du bijou d'Ath-Yenni afin de pérenniser le savoir-faire qui lui est associé.

\section{Fête du bijou d'Ath-Yenni et dynamiques de développement culturel}

3. 1. Les composantes du savoir-faire de bijouterie d'Ath-Yenni : des facteurs clés de valorisation par l'événementiel.

\begin{tabular}{|c|c|c|}
\hline & Ressources individuelles & Ressources collectives \\
\hline $\begin{array}{l}\text { Ressources } \\
\text { matérielles }\end{array}$ & $\begin{array}{l}\text { - Outils de travail. } \\
\text { - Matières premières (argent, corail, } \\
\text { couleurs). } \\
\text { - Ateliers de production. }\end{array}$ & $\begin{array}{l}\text {-Gammes standards de } \\
\text { production (Fibules, bracelets, } \\
\text { bague, etc.) } \\
\text { - Infrastructures patrimoniales } \\
\text { (villages, maisons } \\
\text { traditionnelles). } \\
\text { - Ressources naturelles : Vue sur } \\
\text { le massif du Djurdjura. }\end{array}$ \\
\hline $\begin{array}{c}\text { Ressources } \\
\text { immatérielles }\end{array}$ & $\begin{array}{l}\text { - Le capital cognitif des bijoutiers (la } \\
\text { connaissance): Un savoir-faire } \\
\text { ancestral transmis de parent à } \\
\text { enfant. } \\
\text { - Compétences artistiques. } \\
\text { - Capacité d'adaptation et } \\
\text { d'innovation. }\end{array}$ & $\begin{array}{l}\text { - Capital relationnel. } \\
\text { - Capital social. } \\
\text { - La symbolique ayant trait au } \\
\text { bijou. } \\
\text { - Transmission et apprentissage } \\
\text { au sein de la famille ou du } \\
\text { village auprès des autres } \\
\text { artisans. } \\
\text { - Un label culturel informel: } \\
\text { Lfetta N'At Yenni (Le bijou des } \\
\text { Ath-Yenni. } \\
\text { - L'association des bijoutiers } \\
\text { d'Ath-Yenni. }\end{array}$ \\
\hline
\end{tabular}

Tab. 02 - Les composantes du savoir-faire de bijouterie d'Ath-Yenni. Source : réalisé par nous-mêmes, inspiré de Cominelli (2012).
La réussite de l'événementiel consacré à la promotion du savoir-faire traditionnel ne tient pas uniquement à la valorisation de l'objet patrimonial (produits artisanaux) mais à toutes les ressources constitutives du savoirfaire artisanal. Nous nous intéresserons aux éléments constitutifs du bijou d'Ath-Yenni afin d'identifier ceux que l'événement valorise.

Selon Cominelli (2012), un savoir-faire artisanal est composé de ressources 
matérielles et immatérielles qui sont à la fois spécifiques et génériques. Elles peuvent être le fait de la propriété privée, comme elles peuvent être appropriées collectivement. La figure ci-dessous illustre la composition du savoir-faire de bijouterie d'Ath-Yenni.

La valorisation de la bijouterie d'Ath-Yenni par l'événementiel, devrait se focaliser sur une stratégie intégrée, qui prendrait en compte la totalité de ces composantes qu'elles soient matérielles ou immatérielles.

A travers notre enquête, nous avons déduit que la fête annuelle du bijou, valorise les éléments matériels et immatériels constitutifs du bijou, il s'agit d'un attribut de l'originalité de la fête.

L'élément matériel mis en valeur par la fête renvoi au bijou; les artisans exposent toutes les gammes des produits qu'ils conçoivent, ces dernières sont destinées à la vente car l'événement constitue l'une des occasions de vente par excellence pour les bijoutiers. La présence des touristes locaux et internationaux (diaspora et touristes étrangers) permet aux bijoutiers de rencontrer de nouveaux clients. Les images ci-dessous illustrent une partie de la gamme du bijou d'Ath-Yenni exposée lors de la fête de l'année 2015.

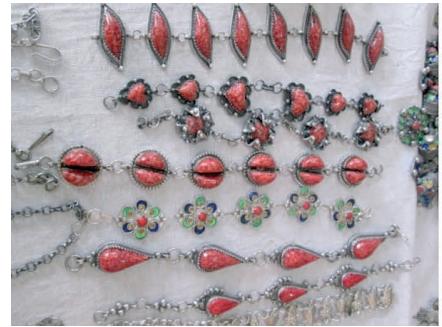

Fot. 3 - Des gourmettes. Source: Photos prises par les auteurs.

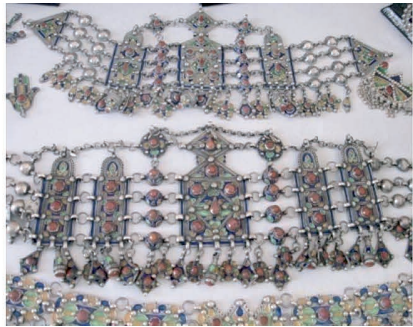

Fot. 4 - Les diadèmes « Tâ̂ssavt». Source: Photos prises par les auteurs.

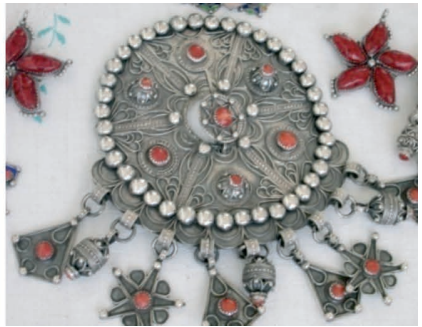

Fot. 5 - Grande fibule « Tafzimt ». Source: Photos prises par les auteurs.

Les éléments immatériels valorisés par la fête sont multiples, d'après les artisans que nous avons interrogés, les artisans diffusent deux types de valeurs à savoir : la valeur artistique et la valeur esthétique.

La finalité patrimoniale tout au long des journées de la fête du bijou n'est pas négligeable, chez les bijoutiers que nous avons enquêté, on trouve des expositions de leurs anciennes collections qui sont soigneusement sauvegardées.

Du point de vue immatériel, l'événementiel permet aux artisans d'exposer la valeur patrimoniale et identitaire de leur métier. Dans ce sens, la fête du bijou traditionnel est l'occasion pour les bijoutiers d'exposer leurs dernières innovations. En effet, parmi les savoir-faire de la wilaya de Tizi-Ouzou, la 
bijouterie d'Ath-Yenni est celle qui a su maîtriser et réussir une dynamique $\mathrm{d}^{\prime}$ innovation tout en sauvegardant le cachet authentique et ancestral du bijou. La transformation du savoir-faire de bijouterie s'est focalisée sur les facteurs clés de sauvegarde de l'identité du bijou à savoir les couleurs, les formes et les symboles. Les images ci-dessous illustres l'effort d'innovation des bijoutiers d'Ath-Yenni.

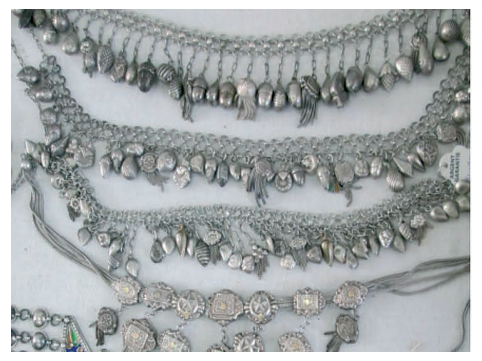

Fot. 6 - Colliers traditionnels. Source: Photos prises par les auteurs.

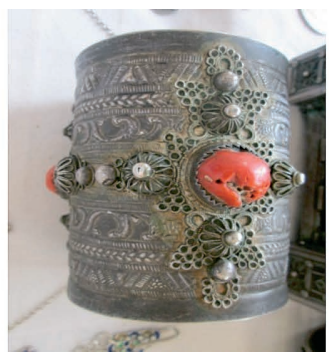

Fot. 7 - Anneaux de chevilles «Axelxal » (Fin du 18ème siècle). Source: Photos prises par les auteurs.

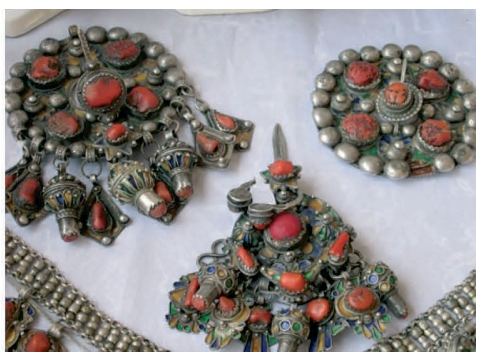

Fot. 8 - Fibules anciennes (fin du 18ème siècle). Source: Photos prises par les auteurs.

L'événementiel constitue pour les artisans interrogés une manière d'évaluer leur réalisation ; tout au long de l'année ils mobilisent leur capital cognitif dans leurs ateliers ou à domicile, leur participation aux festivals et fêtes leur permet d'avoir un retour de la part des clients et visiteurs pour évaluer leurs productions.

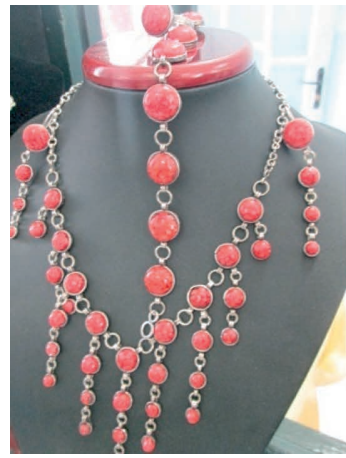

Fot. 9 - Parure moderne en corail. Source: Photos prises par les auteurs.

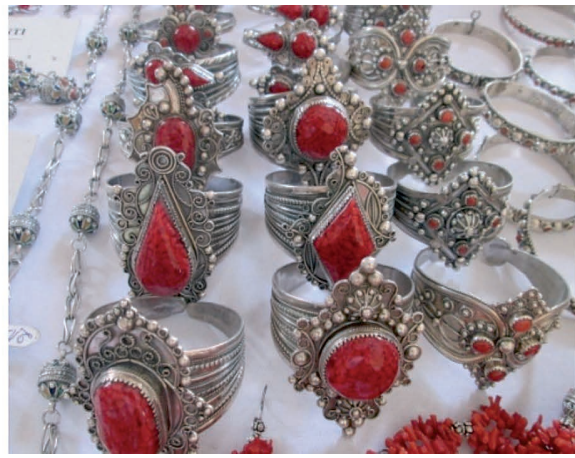

Fot. 10 - Bagues avec des anneaux ouverts. Source: Photos prises par les auteurs.

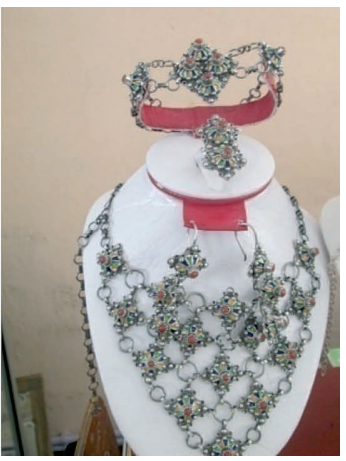

Fot. 11 - Fot. 11 - Parure modernisée. Source: Photos prises par les auteurs.

L'événementiel offre aux artisans l'opportunité de se rencontrer, de partager les informations et de s'inspirer des réalisations des autres bijoutiers. Ceci ouvre la voie aux croisements des connaissances qui renforcent l'esprit 
créatif des artisans, comme il ouvre la voie à des innovations de taille. Les images ci-dessous sont un exemple du croisement de savoir-faire de régions différentes. En effet, un bijoutier d'Ath-Yenni intègre dans sa production le bois d'ébène utilisé par les bijoutiers touaregs et s'est inspiré de leurs motifs tout en sauvegardant le cachet authentique du bijou d'Ath-Yenni. Ce cas est illustré dans les photos ci-dessous:

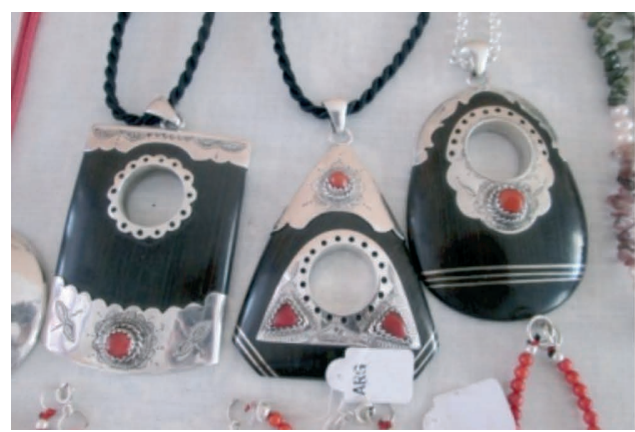

Fot. 12 - Colliers en bois d'ébène, argent et corail. Source: Photos prises par les auteurs.

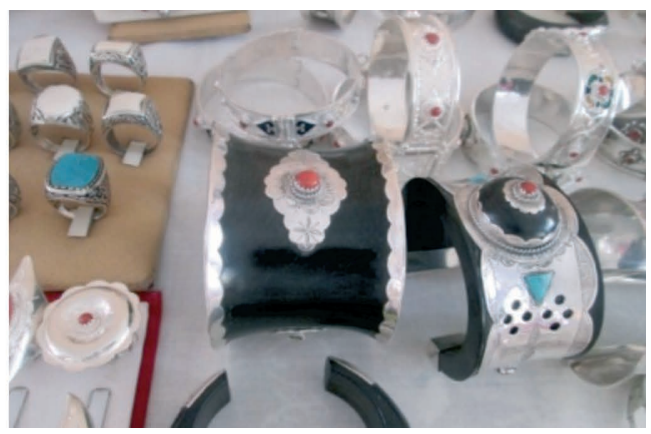

Fot. 13 - Brisselets en bois d'ébène et argent. Source : Photos prises par les auteurs.

Parmi les composantes immatérielles du bijou traditionnel d'Ath-Yenni, le capital social. Ce dernier renvoi à «l'ensemble des ressources actuelles ou potentielles qui sont liées à la possession d'un réseau durable de relations plus moins institutionnalisées d'inter-connaissance et d'interreconnaissance » (Bourdieu, 1980, 2).

Ce Capital est spontanément mobilisé par les artisans d'Ath-Yenni sous formes de rapports sociaux qui se matérialisent par une réciprocité en termes $d$ 'entraide (prêts de matières premières) et d'échange d'informations et d'idées. Il s'agit d'un déterminent territorial de la construction et de la valorisation du savoir-faire de bijouterie. Ces rapports sociaux sont issus de la tradition villageoise kabyle. Notre enquête révèle que ce capital social est fortement mobilisé que ce soit entre les artisans ou entre les communautés villageoises de l'ensemble des villages d'Ath-Yenni qui s'impliquent dans l'organisation de la fête. Celle-ci permet l'apparition des formes de coordinations entre les acteurs des villages d'Ath-Yenni.

Le caractère symbolique du bijou d'Ath-Yenni est l'un des éléments principaux de la construction du savoir-faire de bijouterie ; en effet chaque type de bijou possède une signification et véhicule un message. 
Depuis la transformation de l'usage du bijou, cette symbolique tant à disparaître. La fête du bijou est sensée œuvrer dans le sens de la diffusion de ces significations en tant que patrimoine culturel immatériel.

La valorisation des éléments constitutifs du savoir-faire de bijouterie des Ath-Yenni conditionne une configuration de type " espace-ressource ». Il s'agit de renforcer les liens territoriaux entre le bijou et son territoire (Ath-Yenni). Cette relation consolide l'identité territoriale du bijou et fortifie son ancrage dans le territoire en question comme elle renforce l'attractivité de la région notamment en matière de tourisme en se basant sur un avantage différenciatif à savoir le bijou traditionnel. Le maintien du cachet authentique du bijou par cette fête tient à la consolidation de cette configuration et à la mise en valeur de tous les éléments constitutifs du savoir-faire de bijouterie d'Ath-Yenni.

\section{2. La fête du bijou : un événement opérateur du savoir-faire traditionnel et catalyseur du développement culturel territorial}

François et al (2009) mettent les jalons sur un nouveau concept qui représente le phénomène $\mathrm{d}^{\prime}$ activation de la ressource dans une logique de développement territorial. Il s'agit de " l'opérateur de la ressource ». Ce dernier permet la prise en compte des facteurs internes et externes afin de mobiliser efficacement la ressource et de veiller à sa spécification. Il s'agit de prendre en compte la dépendance existant entre le territoire en tant que terreau de la ressource et le marché en tant que bassin de la demande exprimée vis-à-vis du produit. L'objectif est de développer une spécification propice à un développement culturel local.

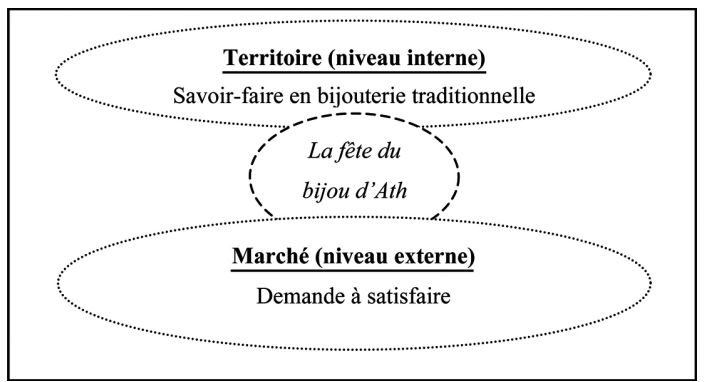

Diagram 1 - Le rôle opérateur de la fête du bijou d'Ath-Yenni. Source : Inspiré de François et al., 2009.
La fête du bijou d'Ath-Yenni joue le rôle d'interface entre le territoire de la région et les acteurs externes, qu'ils s'agissent des clients de la bijouterie ou des visiteurs accueillis par la région. Cette relation est synthétisée à travers du Diagram 1.

En tant qu'opérateur de la ressource patrimoniale, la fête du bijou d'Ath-Yenni renforce

l'attractivité territoriale et la visibilité de la région d'un côté, et émerge des 
activités culturelles de l'autre. Ces dernières concernent l'animation culturelle durant les journées de la fête (galas artistiques, ateliers de divertissement pour les enfants, expositions culturelles diversifiées). Il est admis que l'opérateur de la ressource assure donc la liaison entre la ressource et les acteurs concernés (François et al. 2009). Ainsi la fête du bijou renforce la relation entre les artisans et leur rattachement à leur métier. Il s'agit d'un moment où les bijoutiers évaluent la qualité de leur produit et leur position sur le marché de la bijouterie en argent.

Conclusion

La fête annuelle du bijou d'Ath-Yenni s'inscrit dans une logique de configuration de l'espace-temps, dans le sens où elle assimile au territoire de la région un événement annuel qui renforce l'image et la spécificité culturelle et identitaire de la région.

La fête de bijou d'Ath-Yenni permet une valorisation de la spécificité du bijou traditionnel (qualité, esthétique, authenticité, etc.) et offre une accessibilité de la population à des activités culturelles diversifiées pendant l'évènement. La durabilité de ces retombées est conditionnée par une politique d'investissement culturel. Cette fête implique des acteurs territoriaux qui poursuivent des objectifs multiples et parfois divergents, à cet effet, l'impulsion d'un développement culturel par cet évènement est conditionnée par l'existence d'une gouvernance territoriale afin de réguler l'interrelation des acteurs intervenants.

Le facteur clés de succès de l'événementiel dans la promotion du patrimoine culturel immatériel quelle que soit sa dimension (fête, salon, festival) ne tient pas uniquement à la mise en valeur des produits patrimoniaux (gammes de bijou, de poterie, de vannerie, etc.), cette démarche est réductrice, elle peut appauvrir d'avantage la ressource " savoir-faire traditionnel» et l'inscrire dans une trajectoire de banalisation. Par contre, la réussite de l'événementiel autour des savoir-faire est tributaire de la valorisation des composantes immatérielles du métier. Cette perspective positionnerait le savoir-faire dans une stratégie qui permettrait de pérenniser sa spécificité et d'asseoir une stratégie de développement durable à partir de ce patrimoine vivant.

\section{Références bibliographiques}

BABKINE, A ; ROBIER, A. 2011 « Réussir l'organisation d'un événement », Eyrolles, Edition d'organisation, Paris

BENITO, L. 2002. "Les festivals, entre événement et manifestation culturelle », Evénements, tourisme et loisirs - Cahier Espaces n74, p.24-28

BOURDIEU, P. 1980. «Le capital social : notes provisoires ». Actes de la Recherche 
en Sciences Sociales 31, p. 2-3.

CHOUCHAN. L. et al. (2000), La communication du XXI siècle, Paris, Ed. LPM. COLLETIS, G. ; Pecqueur, P. 2004. "Révélation de ressources spécifiques et coordination située », Economie et Institutions, n 6 et 7, p. 51-74.

COMINELLI, F. 2012, " La tapisserie d'Aubusson inscrite au patrimoine culturel immatériel de l'UNESCO », dans M. Vernières (sous la dir. de), Patrimoine et développement. Etudes pluridisciplinaires, Paris, KARTHALA, p. 53-71.

COURLET, C. 2008. « L'économie territoriale », PUG, Grenoble François.

DI MEO, G. 2001. La géographie en fêtes, Paris, Ed. Ophrys.

DONSIMONI, M. ; KEMMAR, M. ; PERRET-KARNOT. 2010. Les bijoutiers d'Ath

Yenni : Construire une attractivité territoriale sur les savoir-faire artisanaux ancestraux, édition Achab, Tizi-Ouzou, 2010.

FRANÇOIS, H. ; HIRCZAK, M. ; SENIL, N. 2009. « De la ressource à la trajectoire : quelles stratégies de développement territorial », Revue Géographie, Société et Espace, vol 15 (3), p.267-284.

GARAT, I. 2005. " La fête et le festival, éléments de promotion des espaces et représentation d'une société idéale », Annales de géographie, n 643 (3), p. 265-284.

MIRANDA, M. ; CERMAKOVA. 2009. "L'impact de l'événementiel dans le développement touristique des villes: typologies, effets spatiaux et représentation des territoires ", Bulletin de l'Association de géographes français, Association des Géographes Français, 2009, pp.389-397.

PECQUEUR, B. 2006. « Le tournant de l'économie globale », Espaces et sociétés, $\mathrm{n}^{\circ} 124-125$, p. 17-32.

REQUIER-DESJARDINS, M. 2013. « Zones difficiles, politiques publiques et agricultures, cas des zones arides au Maghreb ». Revue des Régions Arides -Numéro spécial- n³1 (2), p. 113-129. 